\title{
The shape of broad-line profiles as diagnostic tool for structure and kinematics of the BLR in AGN
}

\author{
Matthias Zetzl* and Wolfram Kollatschny \\ Institut für Astrophysik, Universität Göttingen, Germany \\ E-mail: zetzleastro.physik.uni-goettingen.de \\ wkollateastro.physik.uni-goettingen.de
}

Broad emission line profiles in AGN can be characterized by their line-width ratios $\mathrm{FWHM} / \sigma_{\text {line }}$. The broad optical and UV emission line profiles - observed in the spectra of Active Galactic Nuclei $(\mathrm{AGN})$ - follow systematic trends in a FWHM/ $\sigma_{\text {line }}$ vs. FWHM diagram. We tried to model these observed characteristics by multiple combinations of different line profile types in a simple way. Finally, we were able to explain all observed trends of the different emission lines in a simple and unique way by Lorentzian profiles that are broadened by rotation. Combinations of other line profiles were less unambiguous and/or could be excluded. To the different emission line regions belong individual turbulent velocities: they range from 400 to $2900 \mathrm{~km} \mathrm{~s}^{-1}$. The observed rotational velocities range from 1000 to $6000 \mathrm{~km} \mathrm{~s}^{-1}$. For the computation of the central black hole masses in AGN on basis of their emission line widths one has first to correct for the contribution of the turbulence onto the widths of the line profile. Otherwise black hole masses are overestimated by a factor of two to ten without correction for the turbulent velocity fraction on the line profile width. Especially the broad UV emission lines have to be corrected for this effect as they are more heavily affected by the additional turbulence component in comparison to the optical lines.

Nuclei of Seyfert galaxies and QSOs - Central engine \& conditions of star formation November 6-8, 2012

Max-Planck-Insitut für Radioastronomie (MPIfR), Bonn, Germany

\footnotetext{
* Speaker.
} 


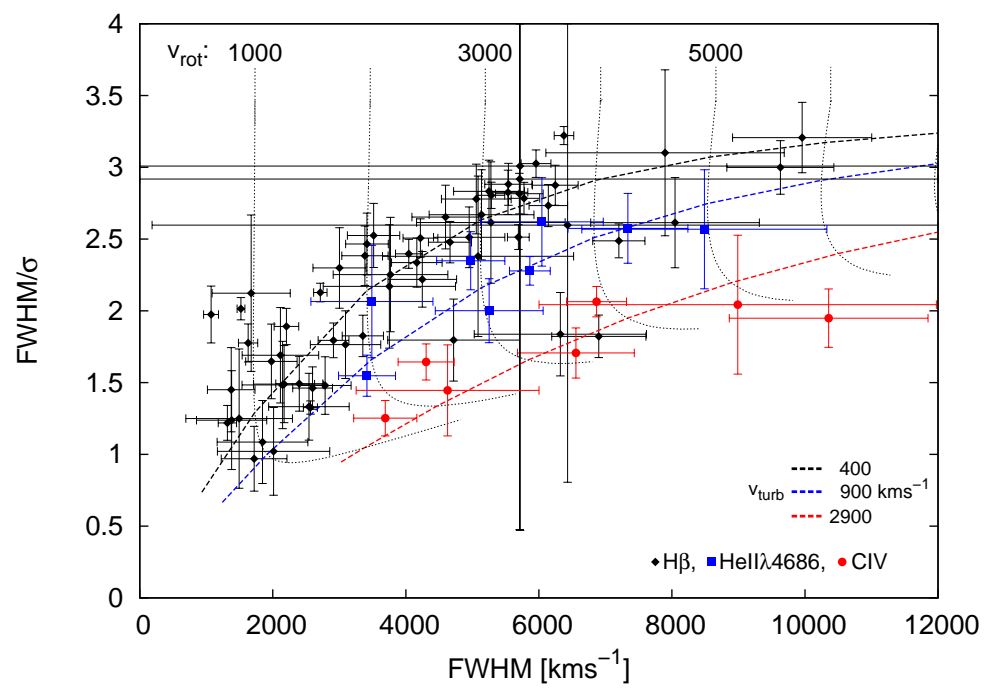

Figure 1: Observed and modeled $\mathrm{H} \beta$ (black diamonds), He II $\lambda 4686$ (blue squares) as well as C IV $\lambda 1549$

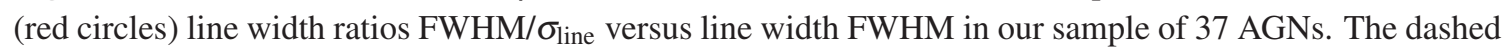
curves show theoretical line width ratios of rotational broadened Lorentzian line profiles; their FWHMs are $400 \mathrm{~km} \mathrm{~s}^{-1}$ (H $\beta$ ), $900 \mathrm{~km} \mathrm{~s}^{-1}$ (He II $\lambda$ 4686) and $2900 \mathrm{~km} \mathrm{~s}^{-1}$ (C IV $\lambda$ 1549) respectively. The associated rotation velocities range from 1000 to $6000 \mathrm{~km} \mathrm{~s}^{-1}$ (curved dotted lines, from left to right, Paper II).

\section{Introduction}

Super-massive black holes in the centers of active galactic nuclei (AGN) are surrounded by an accretion disk. Broad optical/UV emission lines are created by photoionization in the outer region - the broad-line region (BLR) — of the accretion disk. On direct images the BLR is spatially not resolved. However, broad emission line profiles can give us clues to the structure and kinematics of the BLR. We found (Paper I; Paper II) a general trend between the full-width at half maximum (FWHM) and the line width ratio FWHM/ $\sigma_{\text {line }}$ in the broad emission lines of AGN, while different emission lines populate different areas in the FWHM/ $\sigma_{\text {line }}$-vs-FWHM plane (Fig. 1).

\section{The data}

We investigated the profiles of the broad emission lines of 37 AGNs including $\mathrm{H} \beta$, He II $\lambda 4686$ and CIV $\lambda 1549$. These data are based on the largest homogeneous sample on reverberationmapped AGN spectra (Peterson et al., 2004).

\section{Results}

\subsection{Line profile broadening}

We found a basic model that explains the trend found in the FWHM $/ \sigma_{\text {line }}$-vs-FWHM diagram: Intrinsic profiles in AGNs are Lorentzian profiles - caused by turbulence - that are broadened by rotation (Fig. 2). The observed line profiles $S$ can be derived from the convolution of a Lorentzian profile $W$ with an ellipsoidal profile $A$.

$$
S(y)=\int_{-\infty}^{+\infty} W(y-x) A(x) \mathrm{d} x
$$




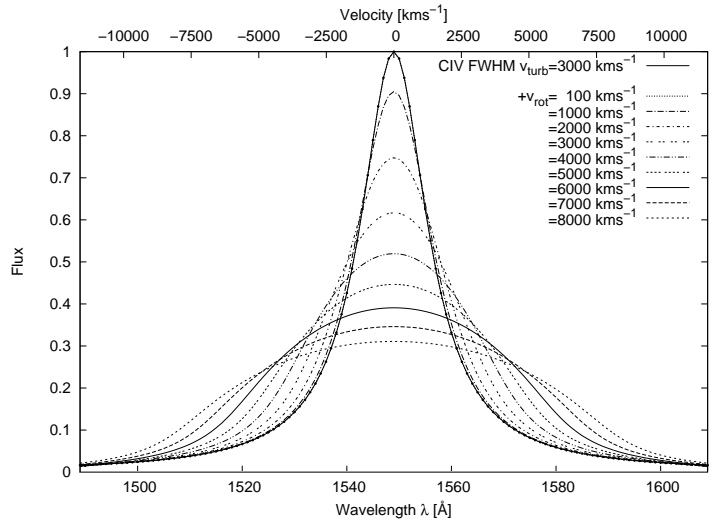

Figure 2: Line broadening of a Lorentzian C IV $\lambda 1549$ profile due to rotation. The rotation velocities range from 100 to $8000 \mathrm{~km} \mathrm{~s}^{-1}$. The intrinsic turbulent velocity of the C IV $\lambda 1549$ line corresponds to $3000 \mathrm{~km} \mathrm{~s}^{-1}$ (Paper II).

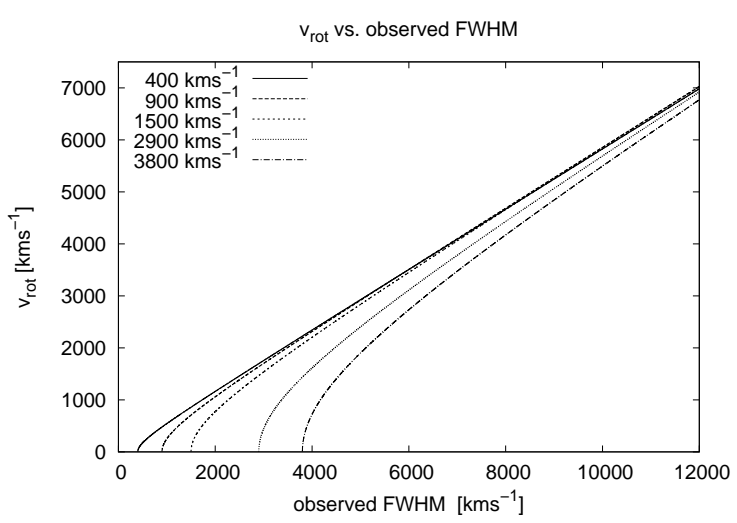

Figure 3: Relation between observed FWHM of the emission line profiles and the related rotational velocity $v_{r o t}$. The relation is shown for intrinsic turbulence profiles with $v_{\text {turb }}$ ranging from $400 \mathrm{~km} \mathrm{~s}^{-1}$ up to $3800 \mathrm{~km} \mathrm{~s}^{-1}$ (Paper II).

The rotational velocity $b=\Delta \lambda / x$ is by definition the half width at zero intensity (HWZI) of an ellipsoidal profile $A$ (Unsoeld, 1955).

$$
A(x)=\frac{2}{\pi} \sqrt{1-x^{2}}
$$

\subsection{BH Masses in AGN}

The black hole masses can be derived from the broad emission line widths under the assumption that the gas dynamics is dominated by the central massive object:

$$
M_{\mathrm{BH}}=f c \tau_{\mathrm{cent}} \Delta v^{2} G^{-1} .
$$

The characteristic distance $c \tau$ of the line emitting regions is known from reverberation mapping studies for the galaxy sample in Fig 1 . The factor $\mathrm{f}$ depends on the kinematics and geometry of the BLR. The line width $\Delta v-$ one is using for calculating the BH mass - should depend on the rotation velocity only and has to be corrected for the contribution of the Lorentzian profile. Figure 3 shows the relation between observed FWHM of the emission line profiles and the related rotational velocity $v_{\text {rot }}$. The relation is shown for intrinsic turbulence profiles with $v_{\text {turb }}$ ranging from $400 \mathrm{~km} \mathrm{~s}^{-1}$ up to $3800 \mathrm{~km} \mathrm{~s}^{-1}$.

\section{Acknowledgments}

This work has been supported by the DFG grant Ko 857/32-1.

\section{References}

Kollatschny, W., \& Zetzl, M. 2011, Nature, 470, 366 (Paper I)

Kollatschny, W., \& Zetzl, M. 2013, A\&A, 549, 100 (Paper II)

Peterson, B. M., Ferrarese, L., Gilbert, K. M., et al. 2004, ApJ, 613, 682

Unsoeld, A. 1955, in 'Physics of stellar atmospheres', Springer publisher 